\title{
Post-surgical outcome for epilepsy associated with type I focal cortical dysplasia subtypes
}

\author{
Samantha L Simpson and Richard A Prayson \\ Department of Anatomic Pathology, Cleveland Clinic Lerner College of Medicine of Case Western Reserve \\ University, Cleveland Clinic, Cleveland, OH, USA
}

\begin{abstract}
Focal cortical dysplasias are a well-recognized cause of medically intractable seizures. The clinical relevance of certain subgroups of the International League Against Epilepsy (ILAE) classification scheme remains to be determined. The aim of the present work is to assess the effect of the focal cortical dysplasia type Ib and Ic histologic subtypes on surgical outcome with respect to seizure frequency. This study also provides an opportunity to compare the predictive value of the ILAE and Palmini et al classification schemes with regard to the type I focal cortical dysplasias. We retrospectively reviewed 91 focal cortical dysplasia patients $(55 \%$ female; median age: 19 years (interquartile range 8-34); median seizure duration: 108 months (interquartile range 36-204)) with chronic epilepsy who underwent surgery. We compared the pathological subtypes, evaluating the patients' post-surgical outcome with respect to seizure frequency according to the Engel's classification and the ILAE outcome classification. Both the ILAE classification scheme and Palmini et al classification scheme were utilized to classify the histologic subtype. Using $\chi^{2}$ and Fisher's exact tests, we compared the postsurgical outcomes among these groups. Of the 91 patients, there were 50 patients with ILAE focal cortical dysplasia type Ib, 41 with ILAE focal cortical dysplasia type Ic, 63 with Palmini et al focal cortical dysplasia type IA, and 28 with Palmini et al focal cortical dysplasia type IB. After surgery, 44 patients (48\%) were seizure-free. Crude analysis revealed no significant difference between patients with subtypes of ILAE focal cortical dysplasia type I or Palmini et al focal cortical dysplasia type I concerning postoperative outcome according to the Engel and ILAE scoring systems on seizure frequency. Our findings revealed no significant difference concerning surgical outcome with respect to seizure frequency for the histologic subtypes of ILAE focal cortical dysplasia type I (Ib vs Ic) or Palmini et al focal cortical dysplasia type I (IA vs IB). In isolation, the histologic subtype of focal cortical dysplasia type I does not appear predictive of postoperative outcome. Modern Pathology (2014) 27, 1455-1460; doi:10.1038/modpathol.2014.64; published online 18 April 2014
\end{abstract}

Keywords: epilepsy; focal cortical dysplasia; ILAE focal cortical dysplasia classification; Palmini focal cortical dysplasia classification; seizures

Focal cortical dysplasias are localized malformations of the cerebral cortex. These lesions are a wellrecognized cause of medically intractable seizures and are associated with derangements of neural circuitries that can be intrinsically epileptogenic. ${ }^{1}$ Approximately $15-25 \%$ of patients seen in epilepsy centers for pharmacoresistant epilepsy have focal cortical dysplasia. ${ }^{2-4}$

There have been numerous published attempts at classification schemes, indicative of disagreement in

Correspondence: Dr RA Prayson, MD, Department of Anatomic Pathology, Cleveland Clinic Lerner College of Medicine of Case Western Reserve University, Cleveland Clinic, 9500 Euclid Avenue, Cleveland, OH 44195, USA.

E-mail: praysor@ccf.org

Received 21 January 2014; accepted 11 March 2014; published online 18 April 2014 definition and approach. ${ }^{5-10}$ In 2004, Palmini et al ${ }^{11}$ published a strictly histopathologic approach that divided focal cortical dysplasias into two major categories. However, apparent inconsistencies in the clinical presentation and variability in imaging characteristics may have, in part, reflected inconsistent histologic diagnoses because of interobserver and intraobserver variabilities. ${ }^{12-13}$ Chamberlain et $a 1^{13}$ found moderate interobserver agreement $(\kappa=0.4968)$ and moderate to very good intraobserver agreement $(\kappa=0.4654-0.8504)$ in assigning histologic subtypes. Focal cortical dysplasia types IA/IB were the least reproducible categories; these subtypes were assigned most frequently in cases without consensus. In 2011, the International League Against Epilepsy (ILAE) proposed a new classification scheme intended to characterize better the clinicopathological focal cortical dysplasia entities. ${ }^{12}$ 
The clinical relevance of certain subgroups of the ILAE classification scheme, most notably in the type I focal cortical dysplasias, remains to be determined. The aim of the present work is to assess the effect of histologic subtype of focal cortical dysplasiaspecifically, types Ib and Ic-on surgical outcome with respect to seizure frequency for patients with medically intractable epilepsy. This study also provides an opportunity to compare the predictive value of the ILAE and Palmini et al classification schemes with regard to the type I focal cortical dysplasias.

\section{Materials and methods}

Institutional Review Board approval was obtained before the commencement of this study. The Anatomic Pathology computer system was reviewed to identify patients with medically intractable epilepsy who underwent surgery between January 2000 and December 2009 and who were diagnosed with focal cortical dysplasia. Patients before 2009 were selected to ensure an adequate follow-up period. All available microscopic slides were reviewed, and using the ILAE classification scheme, focal cortical dysplasia types were assigned. We included for study patients with ILAE focal cortical dysplasia types Ib and Ic. ILAE type Ia cases were extraordinarily rare and, consequently, were excluded from this study. These same cases were also classified according to the Palmini et al classification scheme as type IA or IB.

The ILAE classifies focal cortical dysplasia with abnormal tangential cortical lamination as type $\mathrm{Ib}$ (Figure 1) and focal cortical dysplasia with abnormal radial and tangential cortical lamination as type Ic (Figure 2). The Palmini et al classification divides

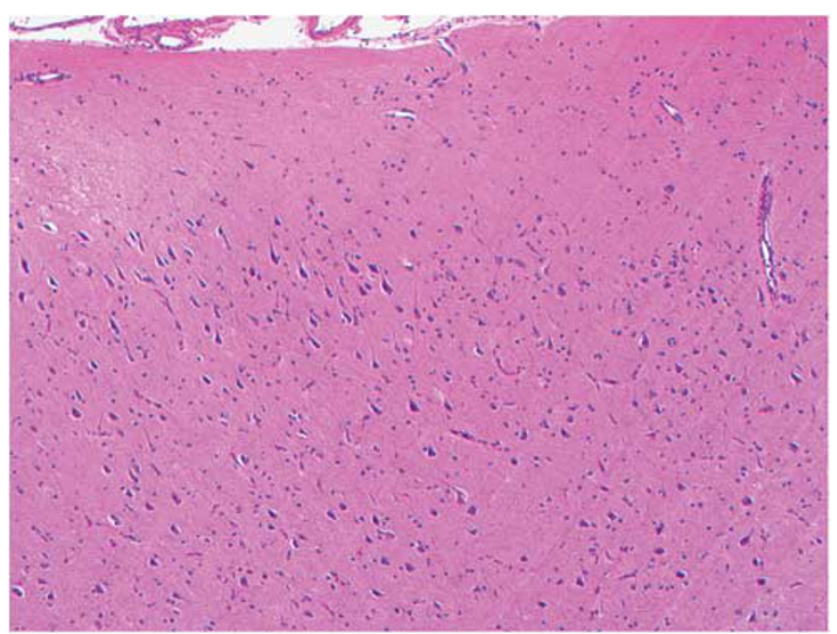

Figure 1 Superficial cortex marked by absence of layer II and a malpositioning of neurons. This pattern would correspond to an International League Against Epilepsy (ILAE) focal cortical dysplasia type Ib or a Palmini et al focal cortical dysplasia type IA. (Hematoxylin and eosin, original magnification is $\times 100$.) type I focal cortical dysplasias into type IA, marked by isolated architectural abnormalities (Figures 1 and 2), and type IB, characterized by architectural abnormalities plus giant or immature neurons (Figure 3). The presence of dysmorphic neurons or balloon cells is characteristic of a type II focal cortical dysplasia in both schemata; these cases were excluded from this study. Cases with coexistent pathologies such as hippocampal sclerosis or tumor (ILAE type III lesions) were also excluded from this study.

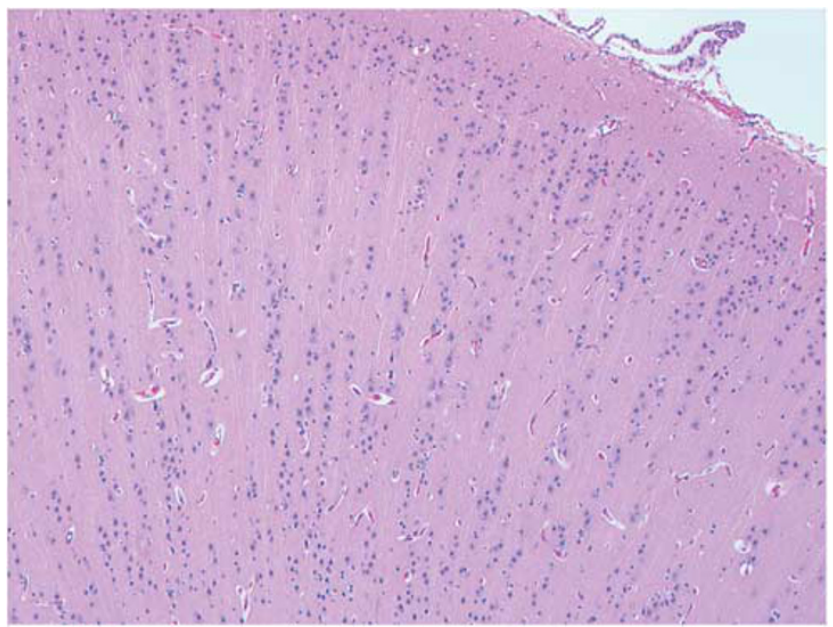

Figure 2 Superficial cortex marked by an abnormal microcolumnar organization in a radial direction. This pattern, in conjugation with a disruption of architecture in a horizontal orientation, corresponds to an International League Against Epilepsy (ILAE) focal cortical dysplasia type Ic or a Palmini et al focal cortical dysplasia type IA. (Hematoxylin and eosin, original magnification is $\times 100$.)

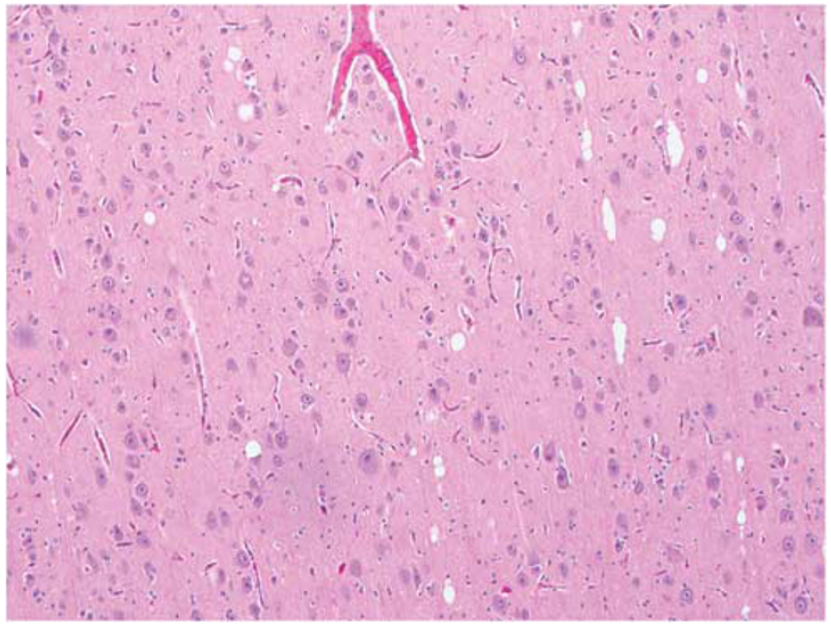

Figure 3 Section from layer III from the cortex marked by neuronal cytomegaly in the background of horizontal disorganization corresponding to International League Against Epilepsy (ILAE) focal cortical dysplasia type Ib or a Palmini et al focal cortical dysplasia type IB. (Hematoxylin and eosin, original magnification is $\times 100$.) 
Sections were routinely generated from formalinfixed, paraffin-embedded tissue from the surgical resection. Specimens were sectioned at $4 \mu \mathrm{m}$ and routinely stained with hematoxylin and eosin. All histologic sections were reviewed, including available immunostains, when performed. All the resections were reviewed. Of the 91 patients who qualified for the study, 28 specimens were totally sampled. The remaining specimens underwent subtotal sampling. The number of slides reviewed on each case ranged from 1 to 22 (mean of 9).

The following patient data were retrospectively collected: gender, age at the time of surgery, duration of epilepsy before surgery, location of resection, history of anti-epileptic drug use, risk factors for epilepsy (eg, head trauma, central nervous system infection, perinatal adverse events, or febrile seizures), and additional surgeries. Imaging studies were reviewed to determine whether the lesion was identified before the first surgery. In patients with a subsequent surgery, the interval to the subsequent surgery and the pathology noted at the time of the subsequent surgery were recorded. Seizure status at most recent follow-up was recorded according to the ILAE's proposed classification of outcome with respect to epileptic seizures ${ }^{14}$ and Engel's classification of postoperative outcome (see Table 1). ${ }^{15}$

We compared the demographic data, using the Student's $t$-test for the continuous variables, age, and seizure duration. We used a $\chi^{2}$ test to compare the categorical variables, gender, and laterality. We used a Fisher's exact test to compare following categorical variables: subsequent additional surgeries and location of the lesion. We calculated the crude outcome analysis with a $\chi^{2}$ or Fisher's exact test. All analyses were conducted using JMP Pro. $P<0.05$ was considered significant.

Table 1 Engel's classification of postoperative outcome and the ILAE proposal for outcome with respect to epileptic seizures

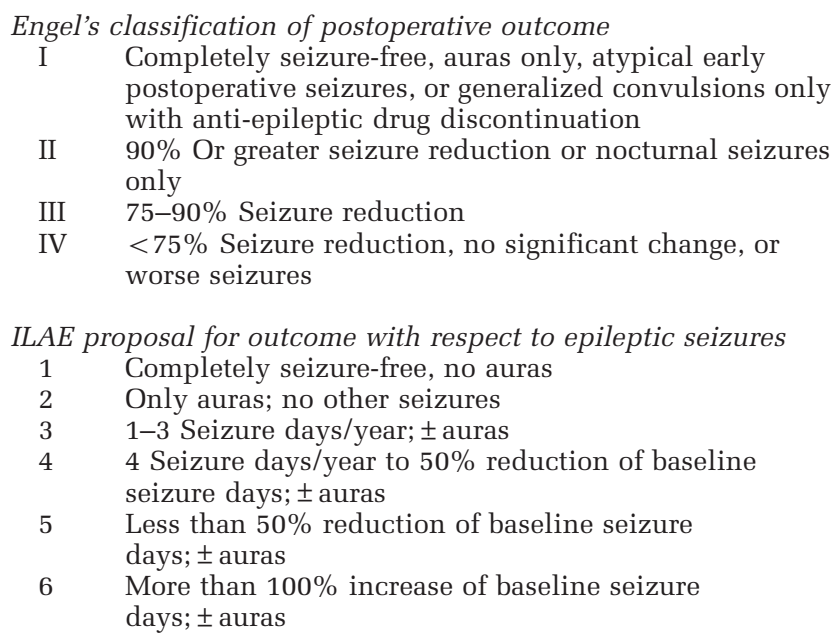

Abbreviation: ILAE, International League Against Epilepsy.

\section{Results}

\section{Patients}

We retrospectively reviewed 91 patients (55\% female; median age at the time of initial surgery: 19 years (8-34 years); median seizure duration: 108 months (36-204 months)).

Of the 91 patients, there were 50 patients with ILAE focal cortical dysplasia type Ib and 41 with ILAE focal cortical dysplasia type Ic. Using the Palmini et al approach, 63 patients were classified with focal cortical dysplasia IA and 28 with focal cortical dysplasia IB. Concomitant pathology findings included subpial gliosis $(n=66)$, fibrosis/ inflammation $(n=54)$, periventricular white matter alterations $(n=40)$, contusions $(n=22)$, hamartia $(n=10)$, calcifications $(n=7)$, vascular sclerosis $(n=1)$, subependymal gliosis $(n=1)$, perivascular lymphocytes $(n=1)$, infarct $(n=1)$, and neuronal heterotopia $(n=1)$. Five patients had nodular heterotopia (ILAE focal cortical dysplasia $\mathrm{Ib}=1$, Ic $=4$; Palmini et al focal cortical dysplasia $\mathrm{IA}=4, \mathrm{IB}=1$ ) and two had polymicrogyria (ILAE Type $\mathrm{Ib}=0$, Ic $=2$; Palmini et al focal cortical dysplasia IA $=1$, $\mathrm{IB}=1$ ). Of the 10 patients who had additional surgeries, two patients had two additional surgeries. Twenty-three patients had a known history of a potential risk factor for epileptic seizures such as head trauma or infection (ILAE focal cortical dysplasia $\mathrm{Ib}=14$, Ic $=9$; Palmini et al $\mathrm{IA}=18$; $\mathrm{IB}=5$ ). All but two cases had identifiable radiographic abnormalities before surgery. Table 2 summarizes the clinicopathologic characteristics of each subgroup.

No other significant differences were found in gender, seizure duration, subsequent surgeries, follow-up interval, or laterality of the lesion between patients with Palmini et al focal cortical dysplasia type IA or type IB. No significant differences were found between patients with ILAE focal cortical dysplasia type Ib and type Ic. Patients with Palmini et al focal cortical dysplasia type IA tended to be older than patients with Palmini et al type IB (Palmini et al type IA median age: 21 years (10-36 years); Palmini et al type IB median age: 14 years (6-22 years)). Second, there were some variations in the location of the lesion between the two groups. Specifically, more patients with Palmini et al focal cortical dysplasia type IA had locations in the temporal lobe than did patients with Palmini et al focal cortical dysplasia type IB (57\% vs $21 \%$ ).

\section{Surgical Outcome in Different Pathologic Subtypes of FCD}

According to the ILAE outcome classification, 44 patients $(49 \%)$ were seizure-free at most recent follow-up after surgery. The median follow-up time for these patients was 63 months (32-93 months). 
Table 2 Summary of patient characteristics

\begin{tabular}{|c|c|c|c|c|c|c|}
\hline & \multicolumn{3}{|c|}{$I L A E$} & \multicolumn{3}{|c|}{ Palmini et al } \\
\hline & $I b(\mathrm{n}=50)$ & Ic $(\mathrm{n}=41)$ & P-value & $I A(\mathrm{n}=63)$ & $I B(\mathrm{n}=28)$ & $\mathrm{P}$-value \\
\hline Age (years) ${ }^{\mathrm{a}}$ & $19(10-35)$ & $18(8-31)$ & 0.58 & $21(10-36)$ & $14(6-22)$ & 0.025 \\
\hline Gender (female) ${ }^{\mathrm{b}}$ & $29(58 \%)$ & $21(51 \%)$ & 0.52 & $34(54 \%)$ & $16(57 \%)$ & 0.78 \\
\hline Seizure duration (months) & $104(47-198)$ & $138(30-207)$ & 0.57 & $114(44-210)$ & $99(31-189)$ & 0.68 \\
\hline Subsequent surgeries ${ }^{b}$ & $4(8 \%)$ & $2(5 \%)$ & & $5(8 \%)$ & $1(4 \%)$ & \\
\hline Interval to subsequent surgeries (months) ${ }^{\mathrm{a}}$ & $42(13-72)$ & $80(10-101)$ & & $46(15-81)$ & $63(4-121)$ & \\
\hline Follow-up interval (months) ${ }^{\mathrm{b}}$ & $65(36-102)$ & $55(19-93)$ & 0.24 & $60(29-87)$ & $55.5(31-104)$ & 0.67 \\
\hline Laterality (left) ${ }^{\mathrm{b}}$ & $26(52 \%)$ & $22(54 \%)$ & 0.87 & $32(51 \%)$ & $16(57 \%)$ & 0.58 \\
\hline Location of the lesion: & & & 0.24 & & & 0.0049 \\
\hline Frontal ${ }^{\mathrm{b}}$ & $24(48 \%)$ & $15(37 \%)$ & & $20(32 \%)$ & $19(68 \%)$ & \\
\hline Temporal $^{\mathrm{b}}$ & $23(46 \%)$ & $19(46 \%)$ & & $36(57 \%)$ & $6(21 \%)$ & \\
\hline Parietal $^{\mathrm{b}}$ & $2(4 \%)$ & $2(5 \%)$ & & $3(5 \%)$ & $1(4 \%)$ & \\
\hline Occipital $^{\mathrm{b}}$ & $1(2 \%)$ & $5(12 \%)$ & & $4(6 \%)$ & $2(7 \%)$ & \\
\hline
\end{tabular}

Abbreviation: ILAE, International League Against Epilepsy.

${ }^{\mathrm{a}}$ Median (interquartile range).

${ }^{\mathrm{b}}$ Number (percentage).

Bolded values were statistically significant at $P<0.05$.

Table 3 Post-surgical outcome related to type I focal cortical dysplasia subtypes

\begin{tabular}{|c|c|c|c|c|c|c|}
\hline & \multicolumn{3}{|c|}{$I L A E$} & \multicolumn{3}{|c|}{ Palmini et al } \\
\hline & $I b(\mathrm{n}=50)$ & Ic $(\mathrm{n}=41)$ & $\mathrm{P}$-value & $I A(\mathrm{n}=63)$ & $I B(\mathrm{n}=28)$ & $\mathrm{P}$-value \\
\hline \multicolumn{7}{|c|}{ Post-surgical outcome according to Engel's classification } \\
\hline Engel outcome I & $28(56 \%)$ & $19(46 \%)$ & 0.36 & $30(48 \%)$ & $17(61 \%)$ & 0.25 \\
\hline Engel outcome I or II & $37(74 \%)$ & $27(66 \%)$ & 0.4 & $43(68 \%)$ & $21(75 \%)$ & 0.52 \\
\hline Engle outcome IV & $3(6 \%)$ & $3(7 \%)$ & 1 & $4(6 \%)$ & $2(7 \%)$ & 1 \\
\hline \multicolumn{7}{|c|}{ Post-surgical outcome according to the ILAE's classification } \\
\hline ILAE outcome 1 or 2 & $26(52 \%)$ & $20(49 \%)$ & 0.76 & $30(48 \%)$ & $16(57 \%)$ & 0.4 \\
\hline ILAE outcome 5 or 6 & $5(10 \%)$ & $5(12 \%)$ & 0.75 & $7(11 \%)$ & $3(11 \%)$ & 1 \\
\hline
\end{tabular}

Abbreviation: ILAE, International League Against Epilepsy.

Total follow-up time for all the 91 patients was 58 months (30-94 months).

Concerning Engel's Classification, the primary outcome of interest was those patients who became seizure-free or had only auras (Type I). A priori secondary end points were those patients who had at least a $90 \%$ reduction in seizure frequency (Types I and II) as well as those who had unfavorable outcomes (Type IV) (Table 3).

The primary outcome with respect to the ILAE post-surgical classification was type 1 or 2 , the categories that are analogous to Engel Type I. A secondary outcome was defined as unfavorable outcomes (ILAE Types 5 and 6).

No statistically significant difference between patients with ILAE focal cortical dysplasia type Ib and patients with ILAE focal cortical dysplasia type Ic concerning postoperative outcome according to the Engel's classification or the ILAE outcome classification was found upon crude analysis (Table 3). Likewise, no statistically significant difference between patients with Palmini et al focal cortical dysplasia type IA and patients with Palmini et al focal cortical dysplasia type IB concerning postoperative outcome according to the Engel's classification or the ILAE outcome classification was found upon crude analysis (Table 3).

\section{Discussion}

To our knowledge, this study is the first to investigate postoperative outcome between the ILAE focal cortical dysplasia type I subtypes. Upon crude analyses, we found no statistically significant difference in post-surgical outcome between patients with subtypes of type I focal cortical dysplasia, whether classified according to the ILAE or Palmini et al scheme. Given the small number of patients who experienced a given outcome, we were unable to perform logistic regression analyses.

According to the Engel's postoperative classification, we identified 47 patients (52\%) who were seizurefree or had only auras at most recent follow-up after 
surgery with a median follow-up time of 61 months (31-94 months). This is similar to the 3-year postoperative outcome of patients with ILAE focal cortical dysplasia type I reported by Fauser et al. ${ }^{16}$ In comparison, Kim et al reported a greater number of patients achieving seizure freedom (66\%). ${ }^{17}$

The ILAE's new classification scheme was intended to better characterize FCD lesions. ${ }^{12}$ This classification system reviewed electroclinical presentations, imaging data, and histopathologic characteristics. Focal cortical dysplasia types I and II are based upon the histopathology of the specimen. Then, this three-tiered classification deviates from a purely histopathologic basis to distinguish isolated focal cortical dysplasia (types I and II) from dysplasias associated with other epileptogenic lesions (Type III). Furthermore, the subgrouping of Type $\mathrm{I}$ is dependent solely upon a somewhat arbitrary division of abnormal radial and/or tangential migration.

Thus, the subtypes for both the Palmini et al and the ILAE classification schemata were developed, at least in part, based upon the histopathologic appearance of the specimens. As the underlying pathomechanisms for focal cortical dysplasias are still poorly understood, perhaps it is unfair to expect the histopathologic subtypes to be predictive of post-surgical outcome.

Our study suggests that the histologic subtype of type I focal cortical dysplasia is not predictive of post-surgical outcome with respect to seizure frequency. The paucity of studies that differentiated among subtypes of type I focal cortical dysplasia when assessing surgical outcome also corroborates this conclusion. Nonetheless, these subtypes may have clinical relevance for other outcome measures that have yet to be explored, such as neuropsychological testing or genetic profiles.

There are, however, some limitations to this study. For instance, the histopathologic classification of these patients was decided by a sole neuropathologist. Given the interobserver variability in applying both the ILAE and the Palmini et al classification systems, ${ }^{13,18}$ it is possible that some cases would have been classified as a different subtype by another pathologist. Second, there was somewhat inconsistent specimen processing. Sixty-three of the ninety-one specimens were subtotally sampled, and the number of slides reviewed on each case ranged from 1 to 22 (mean of 9). A more rigorous scrutiny of these specimens may have provided additional information and changed the histologic classification. Finally, there is variability in the neuroanatomical location of these lesions, resulting in varied surgical techniques to extract the epileptogenic region.

At this juncture, a cogent clinical rationale for subdividing type I focal cortical dysplasia, using either the ILAE or Palmini et al approaches, is lacking. Given the less-than-optimal intraobserver reproducibility among type I focal cortical dysplasia subtypes, one could argue that grouping the lesions together rather than subdividing them may be a more practical approach. Further studies looking at other clinical aspects of type I focal cortical dysplasia subtypes are warranted to clinically validate or invalidate these approaches.

\section{Disclosure/conflict of interest}

The authors declare no conflict of interest.

\section{References}

1 Aronica E, Becker AJ, Spreafico R. Malformations of cortical development. Brain Pathol 2012;22:380-341.

2 Kuzniecky R, Garcia JH, Faught E, et al. Cortical dysplasia in temporal lobe epilepsy: magnetic resonance imaging correlations. Ann Neurol 1991;29: 293-298.

3 Kuzniecky R, Moraustz R, Faught E, et al. Frontal and central lobe focal dysplasia: clinical, EEG and imaging features. Dev Med Child Neurol 1995;37: 159-166.

4 Prayson RA. Diagnostic challenges in the evaluation of chronic epilepsy-related surgical neuropathology. Am J Surg Pathol 2010;34:e1-13.

5 Mischel PS, Nguyen LP, Vinters HV. Cerebral cortical dysplasia associated with pediatric epilepsy. review of neuropathologic features and proposal for a grading system. J Neuropathol Exp Neurol 1995;54: 137-153.

6 Barkovich AJ, Kuzniecky RI, Dobyns WB, et al. A classification scheme for malformations of cortical development. Neuropediatrics 1996;27:59-63.

7 Lawson JA, Birchansky S, Pacheco E, et al. Distinct clinicopathologic subtypes of cortical dysplasia of Taylor. Neurology 2005;64:55-61.

8 Guerrini R, Marini C. Genetic malformations of cortical development. Exp Brain Res 2006;173:322-333.

9 Prayson RA, Estes ML. Cortical dysplasia: a histopathologic study of 52 cases of partial lobectomy in patients with epilepsy. Hum Pathol 1995;26: 493-500.

10 Prayson RA, Spreafico R, Vinters HV. Pathologic characteristics of the cortical dysplasias. Neurosurg Clin North Am 2002;37:17-25.

11 Palmini A, Najm I, Avanzini G, et al. Terminology and classification of the cortical dysplasias. Neurology 2004;62(6 Suppl 3):S2-S8.

12 Blümcke I, Thom M, Aronica E, et al. The clinicopathologic spectrum of focal cortical dysplasias: a consensus classification proposed by an ad hoc Task Force of the ILAE Diagnostic Methods Commission. Epilepsia 2011;52:158-174.

13 Chamberlain WA, Cohen ML, Gyure KA, et al. Interobserver and intraobserver reproducibility in focal cortical dysplasia (malformations of cortical development). Epilepsia 2009;50:2593-2598.

14 Wieser HG, Blume WT, Fish D, et al. Commission on Neurosurgery of the International League Against Epilepsy (ILAE). ILAE Commission Report. Proposal for a new classification of outcome with respect to epileptic seizures following epilepsy surgery. Epilepsia 2001;42:282-286. 
15 Engel J, Van Ness PC, Rasmussen TB, et al. Outcome with respect to epileptic seizures, In: Engel J (ed). Surgical Treatment of the Epilepsies, 2nd edn. Raven Press: New York, NY, USA; 1993, pp 609-621.

16 Fauser S, Essang C, Altenmüller DM, et al. Is there evidence for clinical differences related to the new classification of temporal lobe cortical dysplasia? Epilepsia 2013;54:909-917.
17 Kim DW, Kim S, Park SH, et al. Comparison of MRI features and surgical outcome among the subtypes of focal cortical dysplasia. Seizure 2012;21: 789-794.

18 Coras R, de Boer OJ, Armstrong D, et al. Good interobserver and intraobserver agreement in the evaluation of the new ILAE classification of focal cortical dysplasia. Epilepsia 2012;53:1341-1348. 